\title{
Der Teufel als poetologische Figur in der Erzählung Das Meerwunder des Dresdner Heldenbuchs
}

\section{1}

Anders als in der Hagiographie können monströse und anderweltliche Wesen in der Heldenepik Züge des Teuflischen tragen, ohne dabei eine primär religiöse Bedeutung oder Funktion in den Texten zu haben. ${ }^{1}$ Die Erzählungen integrieren Aspekte christlicher Teufelsvorstellungen, ${ }^{2}$ die aber nicht oder nur marginal auf Logiken oder Praktiken des Glaubens bezogen sind. Das Teuflische ist dann ein Aspekt nichtmenschlicher Figuren, die entweder Teil der Genealogie des Helden sind oder diesem als Antagonisten gegenübertreten. In der Sammlung heldenepischer Texte, die unter dem Namen Dresdner Heldenbuch bekannt ist, findet sich aber auch eine kurze Erzählung, die zwar nach dem literarischen Muster der Heldenepik gestaltet zu sein scheint, in der aber das Teuflische anderweltlicher Figuren in den Mittelpunkt gestellt wird. Diese Erzählung trägt den Titel Das Meerwunder und ist in der Forschung kontrovers diskutiert worden. Ich möchte im Folgenden vorschlagen, den Text poetologisch zu lesen und ihn als religiös perspektivierte Auseinandersetzung mit den heldenepischen Topoi der Konfrontation mit monströsen Gegnern und der Zeugung durch anderweltliche Figuren zu begreifen. Das Meerwunder lässt sich dann als ein Kommentar zu den anderen im Dresdner Heldenbuch versammelten Texten verstehen.

Das Dresdner Heldenbuch (Dresden, Landesbibl., mscr. M 201) enthält Texte der aventiurehaften Dietrichepik: Ortnit und Wolfdietrich, das Eckenlied, den Ro-

1 Vgl. HAMmER, Andreas: Ordnung durch Un-Ordnung. Der Zusammenschluss von Teufel und Monster in der mittelalterlichen Literatur. In: Monströse Ordnungen. Zur Typologie und Ästhetik des Anormalen. Hrsg. von Achim GeISENHANSLÜCKE/Georg MeIN, Bielefeld 2009, S. 209-256, hier S. 233.

2 Vgl. zu Teufelsvorstellungen FrensChKowSKI, Marco/DRASCEK, Daniel: [Art.] Teufel. In: Enzyklopädie des Märchens. Handwörterbuch zur historischen und vergleichenden Erzählforschung, Bd. 13. Hrsg. von Rolf Wilhelm BREDNICH u. a., Berlin, New York 2010, Sp. 383-413, zu christlichen Teufelsvorstellungen des Mittelalters insb. Sp. 384-386; BrENK, Beat: [Art.] Teufel. In: Lexikon der christlichen Ikonographie, Bd. 4. Hrsg. von Engelbert KIRSCHBAUM u. a., Darmstadt 2015 [1968], Sp. 295-300.

Johannes Traulsen, Freie Universität Berlin, Institut für Deutsche und Niederländische Philologie, Habelschwerdter Allee 45, 14195 Berlin, j.traulsen@fu-berlin.de

Ә Open Access. (c) 2021 Johannes Traulsen, publiziert von De Gruyter. (c) BY-NC-ND Dieses Werk ist lizensiert unter einer Creative Commons Namensnennung - Nicht-kommerziell - Keine Bearbeitung 4.0 International Lizenz. https://doi.org/10.1515/9783110667189-005 
sengarten zu Worms, den Sigenot, den Wunderer, den Laurin, die Virginal und das Jüngere Hildebrandslied. Im gesamten Zeitraum des vierzehnten und fünfzehnten Jahrhunderts entstanden ähnliche Zusammenstellungen von Heldendichtungen, doch überliefert sind solche Kompilationen meist erst aus dem letzten Drittel des fünfzehnten Jahrhunderts. ${ }^{3}$ Das Dresdner Heldenbuch ist auf das Jahr $1472 \mathrm{zu}$ datieren und stammt wahrscheinlich aus Nürnberg. ${ }^{4}$ Es handelt sich um die mittelalterliche Sammlung mit den meisten deutschsprachigen heldenepischen Texten überhaupt. Gleichzeitig ist die Dresdner Sammlung das kürzeste der überlieferten Heldenbücher. Brevitas war offenbar ein besonderes Anliegen der Kompilatoren, die in dieser und auch in anderer Hinsicht zum Teil sehr umfangreiche Änderungen gegenüber den Vorlagen vorgenommen haben. Die in das Dresdner Heldenbuch aufgenommenen Texte wurden mit einem Bewusstsein für das Genre der Helden- bzw. Dietrichepik zusammengestellt und lassen deutlich einen Gestaltungswillen der Kompilatoren erkennen. Entsprechend werden die im Dresdner Heldenbuch enthaltenen Erzählungen von der Forschung meist als eigene Fassungen (Dresdner Eckenlied etc.) behandelt. ${ }^{5}$ Kurz nach der Fertigstellung des Manuskripts wurden den Texten des Dresdner Heldenbuchs zusätzlich zu den heldenepischen Erzählungen zwei weitere Texte hinzugefügt, ${ }^{6}$ und zwar die Kurzfassung G des Herzog Ernst und die kurze Erzählung Das Meerwunder, um die es mir im Folgenden gehen wird. ${ }^{7}$ Auch wenn sie nachträglich hinzugefügt worden sind, wurden die eingeschobenen Texte von Schreibern angefertigt, die auch andere Stücke im Dresdner Heldenbuch geschrieben haben. ${ }^{8}$ Sie sind insofern als

3 Vgl. HeINZLE, Joachim: Einführung in die mittelhochdeutsche Dietrichepik, Berlin, New York 1999, S. $43 \mathrm{f}$.

4 Vgl. die Ausführungen von Walter KofLER zur Sprache der Handschrift in: Das Dresdener Heldenbuch und die Bruchstücke des Berlin-Wolfenbütteler Heldenbuchs. Edition und Digitalfaksimile. Hrsg. von Walter KofLER, Stuttgart 2006, S. 20 und S. 50 f. Dieser Ausgabe folgen unter der Sigle DHB im Weiteren auch die Textzitate.

5 Vgl. Millet, Victor: Germanische Heldendichtung. Eine Einführung, Berlin, New York 2008, S. 423-425.

6 Vgl. MilLET, Germanische Heldendichtung, S. 423-425 (Anm. 5).

7 Das Meerwunder ist auch online in einer Edition mit Übersetzung auf den Webseiten der Universität Stuttgart zugänglich (https://www.ilw.uni-stuttgart.de/abteilungen/germanistische-media evistik/forschung/digitale-editionen/meerwunder/meermit.html; 6. September 2019). Die erste Edition des Textes ist: Der Helden Buch in der Ursprache. Zweiter Teil. Hrsg. von Friedrich Heinrich VON DER HAGEN/Alois PRIMISSER, Berlin 1825, S. 222-226. Eine handschriftengetreue Wiedergabe des Textes bietet Fuchs, Edward A. H.: Das Meerwunder. In: Modern Philology 37 (1940), S. $225-240$.

8 Vgl. DHB, S. 12 und S. 53. Der genaue Platz, an dem die Stücke in die Handschrift eingefügt sind, ist insofern nicht aussagekräftig, als für diesen offenbar der jeweilige Platzbedarf und nicht der Inhalt ausschlaggebend war. Vgl. dazu DHB, S. 11. 
zeitgenössisch anzusehen und lassen sich deshalb in einen unmittelbaren Zusammenhang mit den anderen Texten der Kompilation stellen. Außer in der Fassung des Dresdner Heldenbuchs wurde der Stoff von Das Meerwunder von Hans Sachs in zwei Sangsprüchen ${ }^{9}$ verarbeitet.

Das Meerwunder erzählt von einer Königin, die allein am Meer spazieren geht. Dem Wasser entsteigt ein Ungeheuer, von dem die Königin gefangen und vergewaltigt wird. Das Monster ist im Begriff, die Dame umzubringen, als ein Ritter auftaucht und es vertreibt. Er verspricht, das Ereignis geheim zu halten, und geleitet die Königin zurück zu Heim und Ehemann. Doch die Vergewaltigung hat Folgen, denn die Dame ist schwanger und gebiert schließlich ein ganz und gar behaartes Kind mit roten Augen. Der Knabe wird zwar höfisch erzogen, doch mit zwölf Jahren hat er solche Kräfte erreicht, dass er erwachsene Männer verprügelt. Heimlich geht er nachts um, vergewaltigt Jungfrauen und frisst sie hinterher auf. Gegen seinen vermeintlichen Vater und einen Bruder zürnt der wilde Junge und will sie ermorden, um selbst Herrscher zu werden. Das Königspaar und ihr gemeinsamer Sohn kämpfen schließlich in der Thronhalle gegen den teuflischen Bastard, und die Königin verwundet ihn mit Pfeilen so schwer, dass König und Königssohn ihn überwältigen und töten können. Nun berichtet die Königin von der Vergewaltigung. Die Königsfamilie stellt dem Ungeheuer daraufhin eine Falle, indem die Dame erneut ans Meer geht, während König und Königssohn sich versteckt halten. Das Monster wird gestellt und die Königin ersticht es eigenhändig mit dem Schwert. ${ }^{10}$

\section{2}

Die Forschung ist in ihren Versuchen der Deutung von Das Meerwunder sowohl der These nachgegangen, es handle sich um eine heldenepische Erzählung, als auch der Annahme, der Text stehe im Zusammenhang mit religiösen Erzähltraditionen. Die ältere Forschung hat den in Das Meerwunder bearbeiteten Stoff in

9 Vgl. Sachs, Hans: Die Königin mit dem meerwunder. In: Dichtungen von Hans Sachs. Erster Theil. Geistliche und weltliche Lieder. Hrsg. von Karl GoEDEKE, Leipzig 1870 (Deutsche Dichter des sechzehnten Jahrhunderts 4), S. 299-301, und Sachs, Hans: Historia: Königin Deudalinda mit dem meerwunder. In: Hans Sachs, Bd. 16. Hrsg. von Edmund GöTzE, Tübingen 1886 (Bibliothek des litterarischen Vereins CLXXIX), S. 228-232. Beide Texte sind auch Teil des Stuttgarter Editionsprojekts (vgl. Anm. 7). Vgl. zu Hans Sachs' Fassungen auch die Ausführungen unten. 10 Auf eine Ähnlichkeit der Tötung des Meerwunders durch die Königin mit der Tötung Hagens durch Kriemhild weist VooRwinden, Norbert: Das Meerwunder. Heldendichtung oder Märchen? In: ABäG 60/1 (2005), S. 161-182, hier S. 180, hin. 
einen Zusammenhang mit einer merowingischen Abstammungssage zu stellen versucht. ${ }^{11}$ Doch wie bereits Walter HAUG eingewandt hat, ist die Zeugung einer Gründungsfigur durch ein theriomorphes Wesen keineswegs so selten, dass sich die genaue Zuschreibung zu einer bestimmten Dynastie rechtfertigen würde. ${ }^{12}$ HAUG leitet die Handlung von Das Meerwunder eher aus dem Motiv der Zeugung durch ein teuflisches Wesen ab, wie es in der Erzählung von Robert le diable $^{13}$ auftaucht. Robert, der mit dem Herzog der Normandie Robert I. zu identifizieren ist, wurde der Sage nach von einem Teufel gezeugt, konnte sich dann aber von der teuflischen Abstammung durch Buße befreien. Von dieser Logik ausgehend hat HAUG Das Meerwunder als einen Text zu bestimmen versucht, der auf dem Weg zur religiösen Bußlogik der Robert-Geschichte ,steckengeblieben' sei. ${ }^{14}$ Allerdings finden sich in dem Text keine Hinweise, die eine auch nur angedachte Narrativierung religiöser Praktiken wie der Buße nahelegen würden. Über die Herkunft des Stoffes hinaus ist immer wieder die Frage nach der Zugehörigkeit des Textes zur Heldendichtung diskutiert worden. Joachim HEINZLE etwa bezeichnet die Erzählung, ebenso wie den Herzog Ernst G, als ,gattungsfremdes Stück“, ${ }^{15}$ allerdings ohne diese Aussage weiter zu begründen. Stärker differenzierend greift Norbert VOoRWINDEN die Frage nach der Gattung der Erzählung auf, indem er Das Meerwunder als Text an den Grenzen zur Heldendichtung beschreibt, der aber „keine alte Heldensage, sondern eine recht primitive Teufelssage“16 sei. Weiter führt er aus:

Damit gehört das ,Meerwunder` zu jenen Geschichten, die sich im späten Mittelalter formal den Ausläufern der Heldendichtung anschließen, indem sie die für diese übliche Form annehmen und einzelne Namen - Personennamen oder geographische Namen - hinzufügen oder indem sie die Geschichte einem bekannten Helden, vorzugsweise Dietrich von Bern, zuschreiben und so den Anschein erwecken, alte Heldensagen zu überliefern. ${ }^{17}$

11 Vgl. DResCher, Carl: Studien zu Hans Sachs I. Hans Sachs und die Heldensage, Berlin 1890, S. 60-97.

12 Vgl. Haug, Walter: [Art.] Das Meerwunder. In: Die deutsche Literatur des Mittelalters. Verfasserlexikon, Bd. 6. Hrsg. von Kurt RuH u. a., Berlin, New York ${ }^{2} 1987$, Sp. 293-297, hier Sp. $295 f$.

13 Vgl. dazu einführend BERLIOz, Jacques: [Art.] Robert le Diable. In: Lexikon des Mittelalters, Bd. 7, Sp. 914 f. (Brepolis Medieval Encyclopaedias - Lexikon des Mittelalters Online; 8. September 2019).

14 Vgl. Haug, Das Meerwunder, Sp. 296 (Anm. 12).

15 HeINZLE, Einführung in die mittelhochdeutsche Dietrichepik, S. 44 (Anm. 3).

16 Voorwinden: Das Meerwunder, S. 172 (Anm. 10). Mit der Frage der literarischen Gattung des Textes befasst sich auch FichtneR, E. G.: Das Meerwunder: The Progeny of the Monster from the Sea. In: Studia Neophilologica 81/2 (2009), S. 217-232.

17 VooRwinden, Das Meerwunder, S. 172 (Anm. 10). 
VOORWINDEN kommt zu dem Ergebnis, bei Das Meerwunder handle es sich um einen fremden Stoff, der „zu einer Art Heldenlied bearbeitet“"18, also in formaler Hinsicht angepasst wurde. So ergibt sich aus der Forschung der Eindruck einer deutlichen Spannung innerhalb des Textes: Er weist sowohl Elemente weltlicher als auch religiöser Stofftraditionen auf und ist in seiner Form, nicht aber in seinem Gehalt und Handlungsverlauf heldenepischem Erzählen angenähert.

Ich möchte an die Forschung anschließend vorschlagen, dass Das Meerwunder durch die bereits von VoORWINDEN beschriebene Annäherung an die Heldendichtung auch die Funktion eines Kommentars innerhalb des Dresdner Heldenbuchs haben könnte. Die Erzählung greift zwar heldenepische Topoi auf, wird dabei aber nicht zu einem heldenepischen Text. Das betrifft insbesondere die im Zentrum stehende außergewöhnliche Zeugung des Kindes durch das merwunder. ${ }^{19}$ Wie Julia WEITBRECHT gezeigt hat, bleiben solche besonderen Zeugungen auch in den heldenepischen Texten nicht immer unhinterfragt und können dort sowohl stigmatisierend als auch charismatisierend wirken. ${ }^{20}$ Das Meerwunder vereindeutigt das ambivalente Motiv allerdings, indem die Zeugung durch das anderweltliche Wesen keinen Helden hervorbringt, sondern zur Geburt einer teuflischen Kreatur führt und damit den heldenepischen Topos selbst als teuflisch ausweist.

Auch wenn das spätere Teufelskind bereits in der kurzen Vorrede vom Erzähler als solches bezeichnet wird (teuffellische stom, DHB, S. 236, 2,2; „teuflischer Abkömmling“), wird das Ungeheuer aus dem Meer zunächst nicht als Teufel, sondern eben als mer wunder (DHB, S. 236, 2,9) apostrophiert. Die Vorsilbe mer- kann die Fremdheit eines Objekts im Allgemeinen bezeichnen, merwunder können also fremde Kreaturen, ${ }^{21}$ aber auch konkreter Wasserwesen ${ }^{22}$

18 Hier S. 182.

19 Vgl. zur außergewöhnlichen Zeugung in der Heldenepik auch LIENERT, Elisabeth: Exorbitante Helden? Figurendarstellung im mittelhochdeutschen Heldenepos. In: Beiträge zur mediävistischen Erzählforschung 1 (1998), S. 38-63, hier S. 40, die freilich darauf hinweist, dass das Motiv in der deutschsprachigen Heldendichtung nicht sehr verbreitet ist.

20 Vgl. WeitвRеснт, Julia: Genealogie und Exorbitanz. Zeugung und (narrative) Erzeugung von Helden in heldenepischen Texten. In: ZfdA 14 (2012), S. 281-309.

21 Vgl. z. B. Wolfram von Eschenbach: Willehalm. 3., durchgesehene Aufl. Text der Ausgabe von Werner SCHRÖDER. Übersetzung, Vorwort und Register von Dieter KARTSCHOKE, Berlin, New York 2003, 400, V. 26-401, V. 1. Dort wird die Ausstattung von Terramers Heer beschrieben: so und sus gevar / maneger slahte kunder / nach al dem merwunder / hetens uf gemachet, / an koste niht verswachet / nach vogelen und nach tieren (Fremde, verschiedenfarbige und verschiedenartige, zum großen Teil exotische Tiere hatten sie aufgesteckt, die sehr kostbar waren und teils wie Vögel, teils wie Vierfüßler aussahen).

22 Vgl. z. B. Der Stricker: Daniel von dem blühenden Tal. 3., überarb. Aufl. Hrsg. von Michael RESLER, Berlin, Boston 2015 (ATB 92), V. 4279-4286: des quam dâher ein merwîp. / der was aller ihr lîp, / als ein rôse getân / mit der sach man rîten unde gân / ein vil wunderlîchez here. / si was 
sein, die oft halb Mensch und halb Tier sind. ${ }^{23}$ Viele Textbelege dafür finden sich vor allem in heldenepischen Texten und in Artusromanen. ${ }^{24}$ Keineswegs sind merwunder immer bedrohlich, sondern sie können, etwa als Teil der Beschreibung von Enites Sattel in Hartmanns Erec, auch als staunenswert und sehenswürdig gelten. ${ }^{25}$ Merwunder haben damit in der mittelalterlichen Literatur im Allgemeinen einen ambiguen Status. ${ }^{26}$

Die Erzählung Das Meerwunder beschreibt das dem Wasser entsteigende Wesen als monströses Komposit ${ }^{27}$ aus verschiedenen menschlichen und tierischen Elementen:

es het fus als ein fleder maus

und was rauch als ein pere,

ging auf gericht in hohem praus,

recht als es ein mensch were.

es het augen nach falcken art, sein maul was einer spane weit, uber sein prust so ging sein part.

(DHB, S. 236, 3,7-13)

Es hatte Füße wie eine Fledermaus und war zottig wie ein Bär, es lief sehr schnell auf zwei Beinen, ganz als ob es ein Mensch wäre. Es hatte Augen nach Art der Falken, sein Maul war eine Spanne weit, über seine Brust hing sein Bart.

küniginne in dem mere / über diu merwunder, / diu dienten ir alle besunder (Unterdessen kam eine Meerfrau herbei. Deren ganzer Körper war einer Rose gleich, mit dieser Frau sah man ein seltsames Gefolge reiten und gehen. Die war die Königin im Meer und über die Meerwesen, die dienten ihr alle).

23 Vgl. allgemein kulturgeschichtlich einführend SIMEK, Rudolf: Monster im Mittelalter. Die phantastische Welt der Wundervölker und Fabelwesen, Wien, Köln, Weimar 2015, S. 113-131.

24 Vgl. LeXER, Matthias: Mittelhochdeutsches Handwörterbuch. 3 Bde., Bd. 1, Leipzig 1872-1878, Sp. 2119 (http://www.woerterbuchnetz.de/Lexer?lemma=merwunder; 6. September 2019).

25 Vgl. Hartmann von Aue: Erec. Mit einem Abdruck der neuen Wolfenbütteler und Zwettler Erec-Fragmente. Hrsg. von Albrecht LeITZMAnN, fortgeführt von Ludwig WolfF. 7. Aufl. besorgt von Kurt GÄRTNER, Tübingen 2006 (ATB 39), V. 7610-7615: dâ bî daz mer swebete: / dar inne sam er lebete / der visch, bî dem besunder / elliu merwunder / und swaz dâ bûwet des meres grunt. / wer tæte mir der namen kunt? (In der Nähe wogte das Meer. Darin war, als ob er lebte, der Fisch und bei ihm alle Meerwunder und alles, was noch dort am Meeresgrund wohnt. Wer nennt mir ihre Namen?).

26 Vgl. dazu ZinK, Georges: Eckes Kampf mit dem Meerwunder. Zu ,Eckenlied‘ L 52-54. In: Mediævalia litteraria. Festschrift für Helmut de Boor zum 80. Geburtstag. Hrsg. von Ursula HenNiG/Herbert KolB, München 1971, S. 485-492, S. 488 f. Eine ähnliche Handlungskonstellation wie in Das Meerwunder findet sich etwa im Garel vom blühenden Tal des Pleier.

27 Vgl. zu Geschichte und Typologie des Monsters MüHLEMAnN, Simone: [Art.] Monstrum. In: Enzyklopädie des Märchens. Handwörterbuch zur historischen und vergleichenden Erzählforschung, Bd. 9. Hrsg. von Rolf Wilhelm BREDNICH u. a., Berlin, New York 1999, Sp. 823-829. 
Diese Darstellung zielt - ähnlich wie im Fall des Drachen Pfetan im Wigalois $^{28}$ insbesondere auf das Monströse, das zugleich menschlich und tierisch ist. Die Aufzählung der einzelnen Körperteile, die jeweils Tieren zugeordnet werden, lassen die Figur als leiblich korrumpiert, nicht aber als religiös konnotiert erscheinen. Entsprechend wird das Wesen auch als kunder (DHB, S. 236, 3,6), als ,Tier beziehungsweise ,Untier' bezeichnet. Das merwunder wird zunächst als monströses Wesen und „Grenzfigur“29 dargestellt, die aus dem Wasser stammt und der die Königin in der Wildnis begegnet. Damit schließt die Erzählung an weltliche, heldenepische und höfische Traditionen an. Bereits im Akt der Vergewaltigung schlägt dieser nicht-religiöse Entwurf des Wesens jedoch um: Der Erzähler nennt es explizit einen teuffel (DHB, S. 237, 4,2) und die Königin bezeichnet das Ungeheuer als etwas, das als ein teuffel ist gestalt (DHB, S. 237, 4,12; „,wie ein Teufel aussieht“). ${ }^{30}$

Die Konfrontation der Königsfamilie mit dem durch die Vergewaltigung gezeugten Kind wird vollkommen anders narrativ entfaltet: Der Säugling, der von der Königin geboren wird, ist von Anfang an eindeutig als teuflisch dargestellt, was insbesondere an der Reaktion des Königs auf seinen vermeintlichen Sohn erkennbar wird:

Doch wart wachssen der frawen leib, als noch hie tun die zarten weib, wen sie sein schwanger worden. dar nach sie do ein kint gepar: sein haut die was mit schwartzem har geleich der peren orden.

der her und auch die fraw erschrack, do sie das kint an sahen. der her sprach: „was das deuten magk? ob mich got wil verschmahen? des gleich ich nie gesehen han,

28 Vgl. Wirnt von Grafenberg: Wigalois. Text der Ausgabe von J. M. N. KAPTEYN übersetzt, erläutert und mit einem Nachwort versehen von Sabine SEELBACH und Ulrich SEELBACH, Berlin, New York 2005, V. 5084. Vgl. zum Verhältnis von Monstrosität und teuflischen Elementen HAMMER, Ordnung durch Un-Ordnung, S. 236-240 (Anm. 1).

29 GebHaRd, Gunther/GeisLER, Oliver/SchröTER, Steffen: Einleitung, in: Von Monstern und Menschen. Begegnungen der anderen Art in kulturwissenschaftlicher Perspektive. Hrsg. von dens., Bielefeld 2009, S. 9-30, hier S. 14.

30 Dass der Teufel in Tiergestalt erscheint, ist für die mittelalterliche Kunst und Literatur durchaus nicht ungewöhnlich. Vgl. BRENK, [Art.] Teufel, Sp. 298-300 (Anm. 2). Entscheidend ist aber für die folgenden Überlegungen, dass diese Bedeutungsdimension theriomorpher Monstren in der Heldenepik meist nicht aktualisiert wird. 
wan das kint ist rauch als ein per, sein augen rot und schwartze gran.“

(DHB, S. 239, 13,1-13)

Doch begann der Leib der Frau zu wachsen, wie es noch heute den lieblichen Frauen geschieht, wenn sie schwanger geworden sind. Danach gebar sie ein Kind: Seine Haut war mit schwarzem Haar bedeckt, wie es bei Bären der Fall ist. Der Herrscher und die Dame erschraken, als sie das Kind ansahen. Der Herrscher sagte: „Was mag das bedeuten? Ob Gott mich verstoßen will? Gleiches habe ich nie gesehen, denn das Kind ist zottig wie ein Bär, seine Augen sind rot und es hat einen schwarzen Bart.“

Mit der Frage des Königs „Ob mich got will verschmahen?“ (DHB, S. 239, 13,10) und dem Verweis auf die rotglühenden Augen rückt die Erzählung die Teufelhaftigkeit des Neugeborenen in den Mittelpunkt. Darüber hinaus kommt das Wesen nun nicht mehr dem literarischen Topos entsprechend von außen, aus der Wildnis oder Anderwelt, sondern von innen, aus der Königsfamilie heraus. Das von der Königin geborene Kind wird zwar als Teil der Familie anerkannt und aufgezogen: Daz kint zoch man gar lobesam / pis es zu tzwelff jaren kam (S. 239, 14,1f.; „Das Kind wurde auf löbliche Weise erzogen, bis es das zwölfte Lebensjahr erreichte"), erweist sich aber in seinem Verhalten als teuffel (DHB, S. 239, 14,7) und erscheint deshalb als eine Heimsuchung und Prüfung der Königsfamilie. ${ }^{31}$ Das teuflische Wesen entfaltet innerhalb der Gemeinschaft, in die es hineingeboren ist, eine massive destruktive Kraft. Trotz aller guten Erziehung bleibt das von einem nichtmenschlichen Wesen gezeugte Kind ein Teufel und kann nicht in die höfische Welt integriert werden. Es verbreitet ungezügelt Gewalt und stört damit die höfische Gesellschaft empfindlich. Durch den Umstand, dass Ritter und Jungfrauen von dem teuflischen Sprössling umgebracht werden, gerät sogar die genealogische Sicherung des Fortbestands der adligen Kultur in Gefahr. Diese Gefahr kann nicht mit einer heldenhaften Tat, sondern nur durch die gemeinsame Anstrengung der gesamten königlichen Familie überwunden werden.

Es lässt sich festhalten, dass die Erzählung Das Meerwunder im Allgemeinen an heldenepische Erzähltraditionen und Topoi anknüpft, um diese dann aber zu einem weniger konventionellen und - wie noch zu zeigen sein wird religiös konnotierten Ende zu führen. Es stellt sich aber weiterhin die Frage, ob und inwieweit sich auch Bezüge zu den anderen im Dresdener Heldenbuch versammelten Texten erkennen lassen.

31 Zur Überlagerung von Entwürfen monströser und dämonischer bzw. teuflischer Elemente in der religiösen und weltlichen Literatur des Mittelalters vgl. HAMMER, Ordnung durch UnOrdnung, bes. S. 218-221 (Anm. 1). 
Im Dresdner Heldenbuch tauchen noch weitere als merwunder bezeichnete Figuren auf, und zwar im Eckenlied und im Rosengarten. ${ }^{32}$ Im Eckenlied etwa ist der titelgebende Riese Ecke auf der Suche nach Dietrich von Bern und begegnet unterwegs einem Ungeheuer, das zwar nicht dem Meer entsteigt, mit dem Meerwesen in Das Meerwunder aber dennoch die monströse tierähnliche Gestalt teilt: ${ }^{33}$

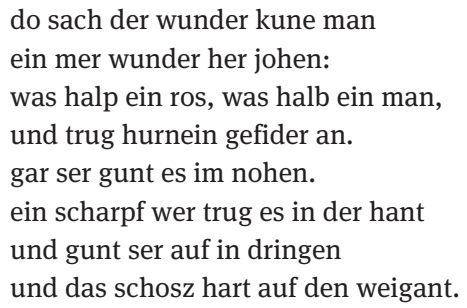

(DHB, S. 136, 48,2-9)

Da sah der sehr kühne Mann ein Meerwunder herbeikommen: Das war halb ein Pferd und halb ein Mann und trug ein Gefieder aus Horn. Es kam sehr nah zu ihm. Eine scharfe Waffe trug es in der Hand, drang mächtig auf ihn ein und schoss hart auf den Helden.

Ecke kämpft mit dem Wesen, besiegt es schließlich und erweist damit seine Fähigkeiten im Kampf, die ihn für die nun folgende Konfrontation mit Dietrich von Bern qualifizieren.

Neben dem monströsen Meerwunder selbst findet sich auch das Motiv der Zeugung einer Figur durch ein Wesen aus einer Anderwelt in weiteren Erzählungen im Dresdner Heldenbuch, und zwar im Ortnit und - im weitesten Sinn auch im Wolfdietrich. Ortnit, dessen Geschichte das Dresdner Heldenbuch eröffnet, ist der uneheliche Sohn des Zwerges Alberich, was dieser ihm allerdings erst offenbart, als Ortnit bereits erwachsen ist:

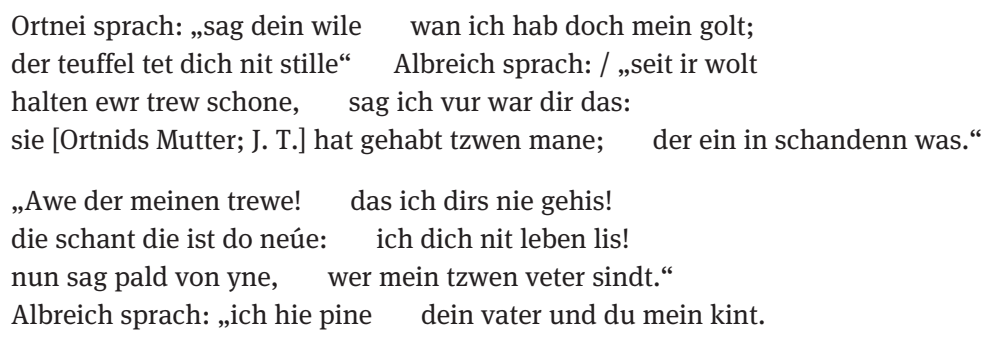

32 Vgl. dazu auch LiEnERT, Elisabeth: Mittelhochdeutsche Heldenepik. Eine Einführung, Berlin 2015 (Grundlagen der Germanistik 58), S. 164.

33 Vgl. zu dieser Episode ZinK, Eckes Kampf mit dem Meerwunder (Anm. 26). 
Zwelf mans sterck und auch grosse $\quad$ - so pin ich neur ein tzwerck:
dar umb ich dir nit gnosse. $\quad$ ich kom aus wildem perg,
das mich do nymant sache, $\quad$ ging in ir kemenot.
den rinck, den du hast dache, $\quad$ den gab ich ir vil drot.“

(DHB, S. 73, 87-89)

Ortnit sagte: „Nenne deinen Wunsch, denn ich habe ja mein Gold; der Teufel lässt dich doch nicht schweigen!“ Alberich sagte: „Da ihr fein treu bleibt, sage ich wahrhaftig dies: Sie hat zwei Männer gehabt, einen davon in Schanden.“ „Oh weh, bei meiner Treue! Hätte ich es dir doch nie gestattet! Die Schande ist jetzt erneuert. Hätte ich dich doch nicht am Leben gelassen! Nun sage mir schnell, wer meine beiden Väter sind.“ Alberich sagte: „Ich bin dein Vater und du mein Kind. Du hast Stärke und Größe von zwölf Männern, während ich nur ein Zwerg bin. Darum ähnele ich dir nicht. Ich kam aus einem wilden Berg. So dass mich niemand sah, ging ich in ihre Kammer. Den Ring, den du versteckt hältst, den gab ich ihr.“

Die Königin und der König hätten keinen Erben zeugen können, so führt Alberich weiter aus, und er sei deshalb ,eingesprungen' und habe die Königin geschwängert, das Kind sei Ortnit. Bekanntlich geht Ortnits Sache nicht gut aus, weil er am Ende der Erzählung von Drachen getötet wird, womit die Handlung zur Wolfdietrich-Sage übergeht. Doch auch der Wolfdietrich verbindet eine Heldengeburt mit einem übernatürlichen Geschehen, stellt dieses aber unter christliche Vorzeichen: Wolfdietrich wird als drittes Kind des nichtchristlichen Herrscherpaars von Konstantinopel geboren. Die Mutter hat eine Vision und lässt das Kind daraufhin von einem Einsiedler taufen. Mit der Taufe geht ein Wunderzeichen einher: Als das Kind ins Wasser getaucht wird, erblickt der Einsiedler viele Lichter darin (vgl. DHB, S. 93, 8), und das Taufhemd wird zur unzerstörbaren Rüstung (vgl. DHB, S. 94, 10). Interessanterweise erscheint auch die dem Kind durch die Taufe gegebene ,christliche Exorbitanz' in der invertierten Perspektive der Nichtchristen als teuflisch, denn auch Wolfdietrich verfügt bereits im Kindesalter über ungeheure Stärke, was die Menschen zu der Annahme verleitet, es wer des teuffels kint (DHB S. 94, 17 „es wäre ein Kind des Teufels“). Der König kann von einem untreuen Vasallen überredet werden, das Kind töten zu lassen, was allerdings nicht durchgeführt wird, Wolfdietrich überlebt und bringt die Mission der Drachentötung, an der Ortnit gescheitert ist, zu Ende. So weisen zumindest zwei Erzählungen des Dresdner Heldenbuchs Darstellungen von Geburt beziehungsweise Taufe auf, die eine nicht- beziehungsweise übermenschliche Komponente haben. An dieses Motiv kann das weiter hinten eingebundene Meerwunder anschließen, allerdings mit der entscheidenden Variation, dass aus der Zeugung kein Held, sondern ein Teufel hervorgeht. ${ }^{34}$

34 Vgl. zur invertierten Logik, dass aus einer durch den Teufel beeinflussten Zeugung der ,wahre Gottesheld“ hervorgeht, den Beitrag von Thomas MÜLLER in diesem Band. 
Weitere Parallelen zu Das Meerwunder bietet im Dresdner Heldenbuch der Wunderer. Zunächst handelt es sich, neben der Virginal, um eine der beiden Enfancen, der Erzählungen von der Kindheit Dietrichs; er ist also - wie Das Meerwunder im Grunde auch - eine Coming-of-Age-Geschichte. Darüber hinaus verbindet sich der Wunderer mit Das Meerwunder über das Motiv der verfolgten Frau $^{35}$ und des ,Jungfrauenfressers‘. Dietrichs Gegner, der titelgebende Wunderer, verfolgt mit seiner Hundemeute eine Jungfrau, die sich der Heirat mit ihm verweigert und die er deshalb fressen will. Doch der Wunderer liefert auch Informationen über seinen Helden Dietrich, der dem Erzähler zufolge trotz seines christlichen Glaubens einer Einflüsterung des Teufels gefolgt ist und deshalb, in einer asketischen Wendung der Handlung, büßen muss:

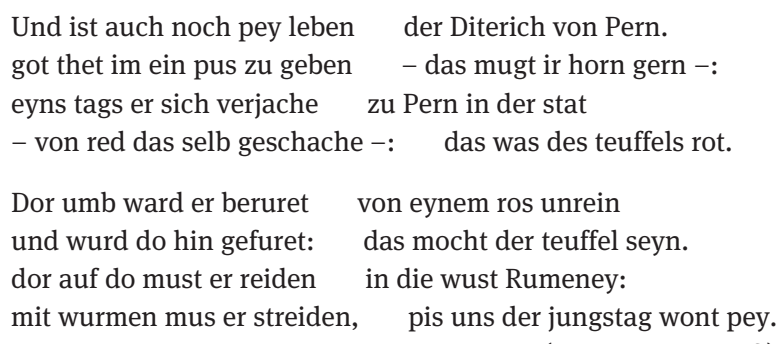

Und noch ist er am Leben, der Dietrich von Bern. Gott hat ihm eine Buße aufgegeben, das sollt ihr gerne anhören: Eines Tages verriet er sich in der Stadt Bern, das geschah durch Worte und war des Teufels Rat. Deshalb wurde er von einem dämonischen Pferd ergriffen und davongetragen: Das war wohl der Teufel. Auf dem musste er in die wilde Rumeney reiten. Mit Drachen muss er kämpfen bis uns der Jüngste Tag gekommen ist.

Dietrichs Buße im Wunderer lässt sich als ein Hinweis auf die nicht ganz unproblematische Stellung der Helden in der Heldenepik zwischen christlichen Transzendenzentwürfen und heroischer Exorbitanz begreifen. ${ }^{36}$ Zwar ist eine Erlösung Dietrichs nach dem Jüngsten Gericht möglich, doch ist die bis dahin zu erbringende Bußleistung des Wüstenlebens bis zum Jüngsten Tag gewaltig. Zudem ist der Text monastisch perspektiviert, indem mit dem Drachenkampf in der Wildnis $^{37}$ nicht nur das Tagesgeschäft der Helden, sondern auch die Antoniuslegende

35 Vgl. DHB, S. 28, Anm. 36.

36 Vgl. auch LIENERT, Mittelhochdeutsche Heldenepik, S. 139 (Anm. 32).

37 Zur Semantik von wust und dem Bezug zur Hagiographie vgl. TRAULSEN, Johannes: Wüste, Wildnis, Einöde. In: Literarische Orte in deutschsprachigen Erzählungen des Mittelalters. Ein Handbuch. Hrsg. von Tilo Renz/Monika HanausKa/Mathias Herweg, Berlin, Boston 2018, S. 598-606. 
angespielt wird. Damit ist eine monastische Perspektive impliziert, denn Antonius gilt als einer der ersten christlichen Eremiten und als Begründer des Mönchtums. Er muss, wie die Vita Antonii des Athanasius von Alexandrien ${ }^{38}$ und die zahlreichen lateinischen und deutschen Fassungen der Legende berichten, ebenfalls in der Wüste gegen dämonische Wesen kämpfen. Einen zweiten Hinweis auf christliche Ideale im Wunderer stellt die verfolgte Jungfrau dar, die sich im Laufe der Handlung als Frau Sælde erweist und ein Keuschheitsgelübde abgelegt hat.

In den Zusammenhang der Konfrontation spezifisch christlicher Entwürfe mit heldenepischen Stoffen fügt sich auch die ebenfalls nachträglich ins Dresdner Heldenbuch eingefügte Fassung G des Herzog Ernst. Die im Spätmittelalter und der Frühen Neuzeit verbreitete Fassung reduziert den Reichsteil auf ein Minimum und verzichtet auf die Magnetberg- und Arimaspi-Episoden. Die Grippia-Episode ist spezifisch umgestaltet: Der Protagonist Ernst rettet die indische Prinzessin aus den Händen der Kranichmenschen, heiratet sie, wird König von Indien, überlässt diese Herrschaft aber seinem Getreuen Wetzel, um selbst schließlich Kaiser zu werden. Der Kampf gegen monströse Wesen einerseits und die Herrschaftsbegründung andererseits scheinen die wichtigsten Anknüpfungspunkte für die Aufnahme des Herzog Ernst $G$ in das Dresdner Heldenbuch gewesen zu sein. Auch hier gibt es also eine Tendenz, den Text heldenepischem Erzählen anzunähern, dabei aber deutlich Position zugunsten des christlichen Helden zu beziehen.

Festzuhalten ist, dass die Auseinandersetzung mit heldenepischen Topoi und christlichen Prinzipien in vielen Texten des Dresdner Heldenbuchs eine Rolle spielt. Dabei kann die religiöse Einhegung der heldenepischen Erzählungen unterschiedlich gestaltet sein und mehr oder weniger eindeutig ausfallen. Die durch eine Engelsvision bewerkstelligte Geburt eines christlichen Helden im Wolfdietrich erscheint als Inversion der Zeugung durch den Zwerg im Ortnit. Im Wunderer muss Dietrich einen durch den Teufel verursachten Fehltritt bis zum Jüngsten Gericht büßen. Der Herzog Ernst $G$ macht einen christlichen Helden und Herrscher, immerhin ist Ernst eigentlich als Kreuzritter unterwegs, zu einer heldenepischen Heroenfigur.

38 Vgl. zur Antoniuslegende GEMEINHARDT, Peter: Antonius. Der erste Mönch. Lebe - Lehre Legende, München 2013. 


\section{3}

In der Forschung wird angenommen, dass Das Meerwunder, anders als die anderen Texte des Dresdner Heldenbuchs, erst im fünfzehnten Jahrhundert entstanden ist $^{39}$ und dass es eine zeitlich nahe, heute verlorene Vorlage für Das Meerwunder gab, die auch die Grundlage für die Bearbeitungen von Hans Sachs bildete. ${ }^{40}$ Die Erzählung ist also mit Blick auf die Tradition, vielleicht auch schon auf Kompilationen wie das Dresdner Heldenbuch entstanden. Die Dresdner Fassung von Das Meerwunder schließt an die heldenepischen Texttraditionen an, indem deren Topoi aufgegriffen werden, etwa die Zeugung durch ein anderweltliches Wesen, die ungeheure Kraft im Kindesalter, die Verfolgung von Jungfrauen und das Menschenfressermotiv. Diese Motivrezeption hat dazu geführt, dass sich die Forschung vor allem mit den heldenepischen Aspekten des Textes befasst hat. Da aber die Motivparallelen einerseits so evident sind, sich andererseits jedoch die Handlung eindeutig gegen heldenepische Logiken entwickelt, ist Das Meerwunder als heldenepischer Text nur unzureichend beschrieben. Vielmehr erscheint es als bewusste Inversion heldenepischen Erzählens und gewinnt dadurch einen reflektierenden und kommentierenden Charakter.

Zunächst fehlt in Das Meerwunder die Position des Helden. Die Zeugung durch das anderweltliche Wesen bringt keinen Heroen, sondern den Antagonisten hervor. Es werden zwar Figuren eingeführt, die als Helden fungieren könnten, doch wird ihnen diese Rolle nicht zugestanden. So verschwindet der Fürst, der das Ungeheuer nach der Vergewaltigung vertreibt, sofort wieder aus der Handlung, ohne weitere Bedeutung zu gewinnen. Die Antagonisten werden gerade nicht im heldenhaften Zweikampf bezwungen, wie in der Dietrichepik (Ecke, Wunderer etc.) üblich. Der König und sein Sohn kämpfen zwar gegen das merwunder und dessen teuflischen Spross, doch sie erscheinen vor allem als Unterstützer der Königin, die in beiden Kämpfen entscheidenden Einfluss nimmt, ohne dabei aber zur Kämpferin zu werden: Auf ihr teuflisches Kind schießt sie während des Kampfes aus der Ferne mit Pfeilen (vgl. DHB, S. 241, 23,9-11); das Ungeheuer aus dem Meer erschlägt sie, als es bereits gefangen ist (vgl. DHB, S. 243, 29,1-13).

Auch eine Aventiurestruktur, wie sie Auseinandersetzungen mit monströsen Gegnern in der aventiurehaften Dietrichepik und der arthurischen Literatur häufig aufweisen, wird in Das Meerwunder konterkariert, obwohl am Anfang des Textes ein abenteure (DHB, S. 137, 1,2) angekündigt wird. Die Monstren agieren im Verborgenen, was der Aventiurelogik widerspricht: Die Königin ver-

39 Vgl. die Einleitung in DHB, S. 37.

40 Vgl. Einleitung in DHB, S. 37. Siehe dazu auch die Ausführungen unten. 
heimlicht die Vergewaltigung durch das merwunder und das Teufelskind verfolgt und frisst die Jungfrauen ebenfalls heimlich. Erst deren Fehlen lässt den König aufmerksam werden (vgl. DHB, S. 239, 15,2-13). Durch das heimliche Agieren aber wird auch der in der Aventiure notwendige Held nicht auf den Plan gerufen. Zwar ist das Motiv angespielt, indem der König nach der Entdeckung der Untaten die Helden seines Reichs aufruft, gegen den Teufel zu kämpfen, doch läuft dieser Handlungsstrang ins Leere, denn alle Recken, die bereit sind, sich der Herausforderung zu stellen, werden sang- und klanglos umgebracht (DHB, S. 140f., 19f.). So bringt die anderweltliche Zeugung im Meerwunder weder einen Helden hervor, noch wird der Kampf mit dem gezeugten Wesen zur Bewährungstat für einen Helden. Vielmehr wird die in der Heldenepik topische Konfrontation mit einem anderweltlichen Gegner in die Abwehr eines teuflischen Wesens übergeleitet, das nicht durch die Tat eines einzelnen Helden, sondern nur durch die Gemeinschaft der Familie überwunden werden kann. Der heroische Kampf ist zugunsten einer Auseinandersetzung mit dem von innen kommenden Bösen variiert. Bereits in der ersten Strophe von Das Meerwunder wird zudem betont, dass sich das Agieren des teuflischen Widersachers insbesondere gegen Frauen richtet: [E]r trug der reinen frawen has (DHB, S. 236, 1,11; „Er hasste die keuschen Damen“). Damit rückt ein Gegenüber von Teufel und Jungfrau in den Mittelpunkt, das seine Entsprechung nicht in der Heldenepik, sondern in der Hagiographie, etwa in der Konfrontation der Heiligen Margareta mit dem Drachen hat. ${ }^{41}$ Die Königin erkennt zudem bereits in dem Wesen aus dem Meer das Teuflische, ${ }^{42}$ womit sie das Dämonische des gezeugten Kindes vorwegnimmt. Da die Königin auch im Kampf gegen die Monstren eine zentrale Rolle spielt, lässt sich hier eine Verschiebung gegenüber den heldenepischen Narrativen beobachten: Den männlichen Helden wird eine weibliche Figur gegenübergestellt, die sich einerseits in ihrem Widerstand gegen den Angreifer auf Gott bezieht (vgl. O her, nun pis mein schirm [und] schilt, DHB, S. 237, 4,9; „Oh Herr, sei jetzt mein Schirm und Schild“) und sich andererseits in der Gemeinschaft selbst am Kampf beteiligt.

41 Vgl. etwa die entsprechende Darstellung bei Jacobus de Voragine: Legenda aurea / Goldene Legende, Bd. 2. Hrsg. von Bruno W. HÄUPTLI, Freiburg i. Br. 2014 (Fontes Christiani Sonderband), S. 1218.

42 Sie benennt das Wesen vielfach entsprechend. Vgl. DHB, S. 237, 4,12: als ein teuffel [. . .] gestalt; 5,6: teuffels pilde; 6,9: teuffel; 6,11: teuffellische pild; 7,11: teuffel etc. 


\section{4}

Sechzehn Jahre vor dem Dresdner Heldenbuch wird Thürings von Ringoltingen Melusine fertiggestellt. Abschriften dieses Textes könnten schon bald in Nürnberg kursiert und vielleicht auch den Kompilatoren des Dresdner Heldenbuchs vorgelegen haben. In der Melusine erscheint die Zeugung mit einem anderweltlichen Partner ebenso wie in den heldenepischen Texten als bedrohlich, aber zugleich als produktiv und genealogiestiftend. ${ }^{43}$ Betrachtet man Das Meerwunder vor dem Hintergrund dieser andauernden Attraktivität des literarischen Motivs der Zeugung durch ein anderweltliches Wesen, so lässt es sich als Kommentar im Hinblick auf den literarischen Diskurs, aber auch auf die Sammlung des Dresdner Heldenbuchs verstehen. Ein heldenepischer Topos wird in Das Meerwunder nachdrücklich pejorisiert und damit werden die heldenepischen Stoffe selbst als fragwürdig ausgestellt.

Ein kontrastierender Vergleich mit den Sangspruch-Bearbeitungen des Stoffes durch Hans Sachs vermag die Deutung des Dresdner Meerwunders als poetologische Erzählung weiter zu schärfen. So weist der Spruch Historia: Königin Deudalinda mit dem meerwunder ${ }^{44}$ gerade die metapoetisch zu begreifenden Aspekte nicht auf. Im Sangspruch rückt der König so weit, wie es die Kürze der Erzählung erlaubt, in die Rolle des Helden. Das Teufelskind tötet und frisst keine Jungfrauen, sondern nur ,etliche Männer‘, woraufhin es von König und Königssohn konfrontiert wird. Die folgende Tötung des Meerwesens wird mit der Haltung des Königs begründet: Doch hett er [der König, J. T.] lust auch mit gefahr, / Dises meerwunder selbst zu sehen, / Von dem diser grewl war geschehen (S. 231, 17-20; „Doch begehrte er trotz der Gefahr, dieses Meerwunder selbst zu sehen, von dem die Gräuel gekommen waren“), heißt es bei Sachs, der mit der ,Abenteuerlust` des Königs eine Aventiure-Logik impliziert. Das Epimythion des Sachs'schen Textes (vgl. S. 232, 6-31) macht die Erzählung zu einem Exempel für Frauen: Man könne aus der Geschichte lernen, dass Frauen die Einsamkeit meiden und sich stets in Gesellschaft aufhalten sollen, womit die Verantwortung für die Ereignisse auf die weibliche Figur verlagert wird.

Christian KIENING hat mit Blick auf die oben beschriebene anderweltliche Zeugung im Ortnit betont, dass sich aus der Fragilität, die sich aus dem Fehlen eines normgerecht gezeugten Erben ergibt, eine Poetizität nährt. ${ }^{45}$ Das Meerwunder greift im Gegensatz dazu den literarischen Topos zwar auf, nicht aber um ihn auszuerzählen, sondern um ihn kritisch zu reflektieren. Von der ander-

43 Vgl. KIEnING, Christian: Unheilige Familien. Sinnmuster mittelalterlichen Erzählens, Würzburg 2009 (Philologie der Kultur 1), S. 191.

44 Siehe Anm. 9.

45 KIENING, Unheilige Familien, S. 30. 
weltlichen Zeugung geht in der Handlung keine produktive Kraft aus, die einen Helden oder gar ein ganzes Geschlecht hervorbringt, sondern ein destruktives Moment. Entsprechend lässt sich auch der Umgang mit dem Topos deuten: Das heldenepische Motiv wird nicht fortgeschrieben und entfaltet insofern keine poetische Produktivität, sondern wird an ein Ende geführt, an dem es als teuflisch erkennbar ist. Damit ergibt sich eine poetologische Dimension im Hinblick auf die heldenepische Erzähltradition, deren Sammlung und Fortschreibung das Dresdner Heldenbuch dient. Mit diesem reflexiven Charakter fügt sich Das Meerwunder trotz allem gut zu den im Dresdner Heldenbuch versammelten Texten, denn von diesen lassen viele ebenfalls eine kritische Auseinandersetzung der Bearbeiter mit den heldenepischen Topoi derselben erkennen.

Natürlich sind die Epimythien der mittelalterlichen Literatur nicht über die Gebühr zu belasten, aber es ist denkbar, dass der Erzählerrat dopei so nemet lere, / das man in solchen dingen sei / verschwigen und getrewe (DHB, S. 243, 31,6-8; „davon sollt ihr lernen, dass man in solchen Dingen treu und verschwiegen sein soll“) sich nicht auf die Vergewaltigung bezieht, sondern auf das Erzählen von den anderweltlichen Wesen selbst, denn - so zeigt es jedenfalls Das Meerwunder der heldenepische Topos zeugt den Teufel. 